\title{
Thermal Orientation Evaluation for Carbon-fiber-reinforced Plastic by Periodic Heating Method
}

\author{
Sho Nagata, Shugo Miyake, and Junichi Ozaki \\ Department of Mechanical Engineering, Kobe City College of Technology \\ 8-3 Gakuenhigashi-machi, Kobe 651-2194, Japan
}

(Received September 19, 2018; accepted November 20, 2018)

Keywords: periodic heating, thermal diffusivity, heat propagation, phase lag, CFRP

Since the thermal anisotropy of carbon-fiber-reinforced plastic (CFRP) depends on the direction of the carbon fibers, it is important to understand the thermal characteristics of CFRP in a three-dimensional thermal propagation. To reveal the relationship between the direction of the carbon fibers and the preferred thermal orientation, we developed a method of estimating thermal anisotropy by analyzing the dependence of measured phase lags on the distance from a heating spot, on the basis of a periodical heating method. Measurement results of the CFRP samples with different fiber orientations in the thickness direction, fabricated by hot pressing, showed a mismatch between the fiber and thermal orientations. It was found that the fiber orientation is not necessarily in conformity with the thermal orientation. The proposed method might be effective for evaluating the thermal anisotropy of CFRP.

\section{Introduction}

Nowadays, because of its very light weight and high fracture strength, carbon-fiberreinforced plastic (CFRP) is used as a new functional material in various fields such as the automotive and aeronautical fields. ${ }^{(1-9)}$ Hence, it is important to evaluate the thermal characteristics of CFRP to advance its practical application. ${ }^{(10,11)}$ CFRP shows characteristic physical properties that depend on the type of matrix resin and molding conditions such as pressing temperature and forming pressure. ${ }^{(2,3)}$ Moreover, its physical properties show large anisotropy in the direction of the carbon fibers, ${ }^{(12,13)}$ thus, CFRP has a thermal anisotropy due to the large difference between the thermal conductivities of the carbon fiber and the matrix resin and also a thermal resistance between their interface. However, the effect of the orientation of the carbon fibers on heat propagation is not very clear. In this study, to understand the behavior of heat propagation inside the CFRP, we investigated the relationship between the direction of the carbon fibers and the characteristics of heat propagation by a laser spot periodic heating method.

The laser spot periodic heating method, which is one of the evaluation methods for anisotropic materials, is known as a highly accurate and noncontact thermal diffusivity

*Corresponding author: e-mail: miyake@kobe-kosen.ac.jp

https://doi.org/10.18494/SAM.2019.2136 
evaluation method. ${ }^{(14-16)}$ In this method, by positioning heating and detecting regions on the front and back surfaces of a sample, we can obtain three-dimensional thermal information. In this paper, as a fundamental research, we report on a thermal anisotropy evaluation method with periodic heating for CFRP.

\section{Experiments}

\subsection{Principle of periodic heating method and equipment}

First, we prepared CFRP samples with a thermal anisotropy depending on the carbon fiber direction and cut the samples at several fiber orientations. We conducted a measurement experiment for these samples with periodic heating. Figure 1 shows a schematic of periodic heating in the in-plane direction and its cross-sectional view. If there is a periodic heating point source on a sample surface, by considering a sample to be an isotropic infinite continuous medium with thermal diffusivity $\alpha$ and specific heat capacity $C$, the alternating current component $T_{0}(x, \mu, t)$ of the temperature is determined to $\mathrm{be}^{(14-17)}$

$$
T_{0}(x, \mu, t)=\frac{l_{0}}{4 \pi x C \alpha} e^{-\frac{x}{\mu}+i\left(\omega t-\frac{x}{\mu}\right)},
$$

where $l_{0}$ is the initial power of the heating laser beam, $x$ is the distance from the point heat source to the detection point, $\mu$ is the thermal diffusion length, and the temperature wave number $k$ is defined as

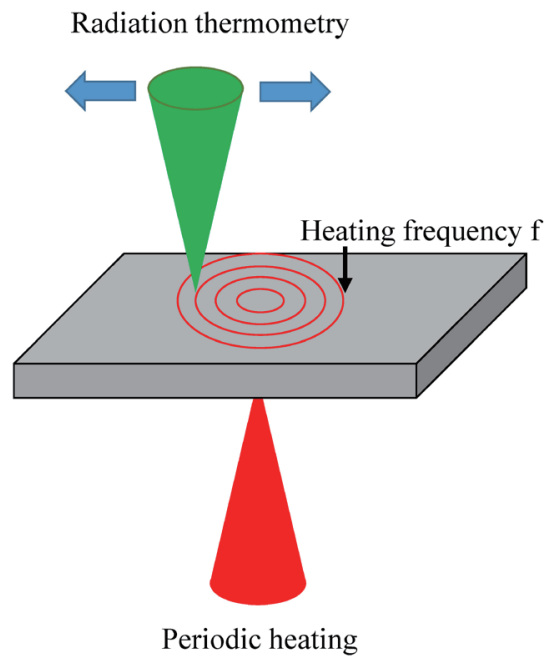

(a)

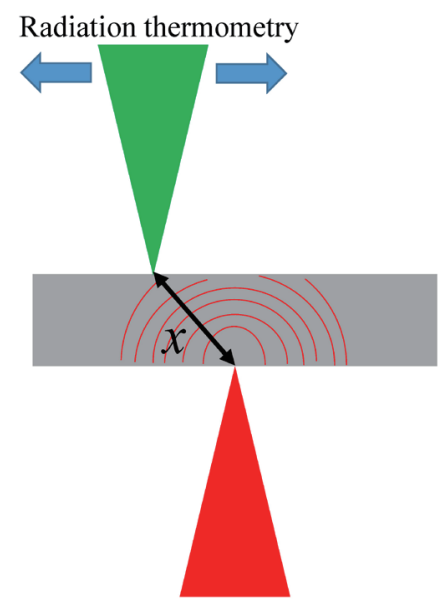

Periodic heating

(b)

Fig. 1. (Color online) Schematic of (a) periodic heating and (b) temperature wave into sample in in-plane measurement. 


$$
k=\left(\frac{\omega}{2 \alpha}\right)^{1 / 2}=\left(\frac{\pi f}{\alpha}\right)^{1 / 2}=\mu^{-1}
$$

Also, from Eqs. (1) and (2), the phase lag $\varphi$ is defined as

$$
\varphi=k x=\left(\frac{\pi f}{\alpha}\right)^{1 / 2} x
$$

Although $\varphi$ is expressed as a function of $x, f$, and $\alpha$, measurement conditions are limited by the shape and thermal properties of the sample in actual measurements. Thus, it is important to determine measurement conditions carefully by considering not only the sample thickness and thermal diffusivity but also the appropriate frequency range of heating laser intensity modulation.

The thermal diffusivity in the out-of-plane direction is determined by scanning the heating frequency for heating in alignment with the detection point in the thickness direction. From Eq. (3), when the heating frequency is given an appropriate range in out-of-plane measurements, the square root of the modulation heating frequency $f^{0.5}$ and the phase lag $\varphi$ show a proportional relationship. Therefore, the measurement result is fitted by linear regression, and the thermal diffusivity is calculated from the linear slope. The thermal diffusivity in the in-plane direction measurement is determined by scanning the detector in one direction with a constant heating frequency. The detected phase lag $\varphi$ is the difference in the phase of the temperature wave from the heating laser at each point in in-plane measurement. That is, the minimum phase lag point indicates the position where the transmission of heat propagation occurs at the earliest point in the scanning position range.

A thermowave analyzer (TA, Bethel Co., Ltd.) based on the periodic heating method was used in this study. A block diagram of the TA is shown in Fig. 2. The intensity of the heating laser emitted from the laser diode is modulated using a function generator. The modulated laser beam irradiates the back surface of the sample via an optical fiber. The temperature response of the front surface of the sample is detected using an infrared semiconductor detector and input to the lock-in amplifier as a detection signal. The lock-in amplifier analyzes the phase lag from the reference and detection signals, and outputs the result to a personal computer.

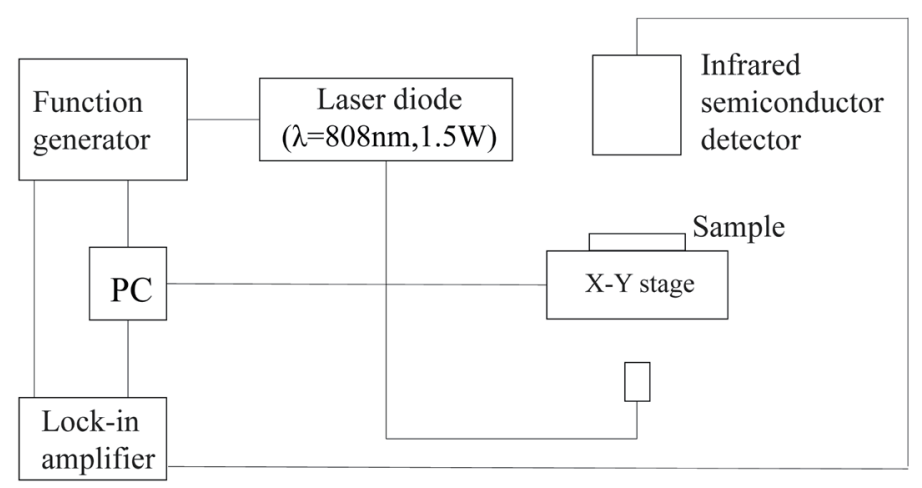

Fig. 2. Block diagram of the thermowave analyzer. 


\subsection{Preparation of CFRP samples}

CFRP samples were fabricated by a hot press method. In this experiment, the carbon fiber used was $7 \mu \mathrm{m}$ in diameter and contained 3000 filaments, and polyamide was used as the matrix material. To make the fibers unidirectional, the carbon fibers and matrix were inserted in a metal mold with an area of $120 \times 100 \mathrm{~mm}^{2}$ and a thickness of $38 \mathrm{~mm}$. A heater was attached to a pressing machine, and molding was carried out by pressing the metal mold. The molding temperature was set at $523 \mathrm{~K}$ considering the melting point of polyamide $(503 \mathrm{~K})$ and the pressing pressure was $2.4 \mathrm{MPa}^{(18)}$ The molded CFRP was cut using a fine cutting machine to obtain samples with various orientations in the range from 0 to $90^{\circ}$, an area of approximately $4 \times 20 \mathrm{~mm}^{2}$, and a thickness of $1 \mathrm{~mm}$.

To confirm the fiber direction of each sample, cross-sectional observation was carried out by SEM. Table 1 shows representative SEM images obtained at carbon fiber angles of 90 and $40^{\circ}$. Carbon fibers are in parallel alignment and have a high density. The carbon fiber angles of each sample were measured from these SEM images to be $0,6,20,40,55,70,76$, and $90^{\circ}$.

\subsection{Experimental procedure}

We conducted phase lag measurements in in-plane and out-of-plane directions by periodic heating, and calculated the thermal diffusivity and heat propagation angle. Figure 3 shows a schematic of the layout of the phase lag measurement in the in-plane direction and the relationship between the carbon fiber angle $\theta$ and the heat propagation angle $\theta>$ in the CFRP. When the formed angles $\theta$ and $\theta>$ are $90^{\circ}$, as shown in Fig. 3(a), the fastest phase lag point is at the 0 position of the detection axis. However, as shown in Fig. 3(b), the formed angles of $\theta$ and $\theta>$ exhibit different values. In particular, when $\theta$ is 90 or $0^{\circ}$, a parabolic distribution of the phase lag is obtained, such as that of an isotropic material, as shown in Fig. 3(a). Thus, these results indicate that the heating and detection axes are aligned and have become coaxial, such that the minimum phase lag point is at the 0 position on the detection axis. In contrast, if the heat propagation angle $\theta^{\prime}$ does not correspond to the carbon fiber angle $\theta$, the minimum phase lag point shifts to the direction in which the carbon fiber is inclined, as shown in Fig. 3(b). Here, the distance $r$ at the minimum phase lag point on the detection axis in Fig. 4(b) is defined as

Table 1

Observation of part of the cross section of the CFRP at carbon fiber angles of 90 and $40^{\circ}$ by SEM.

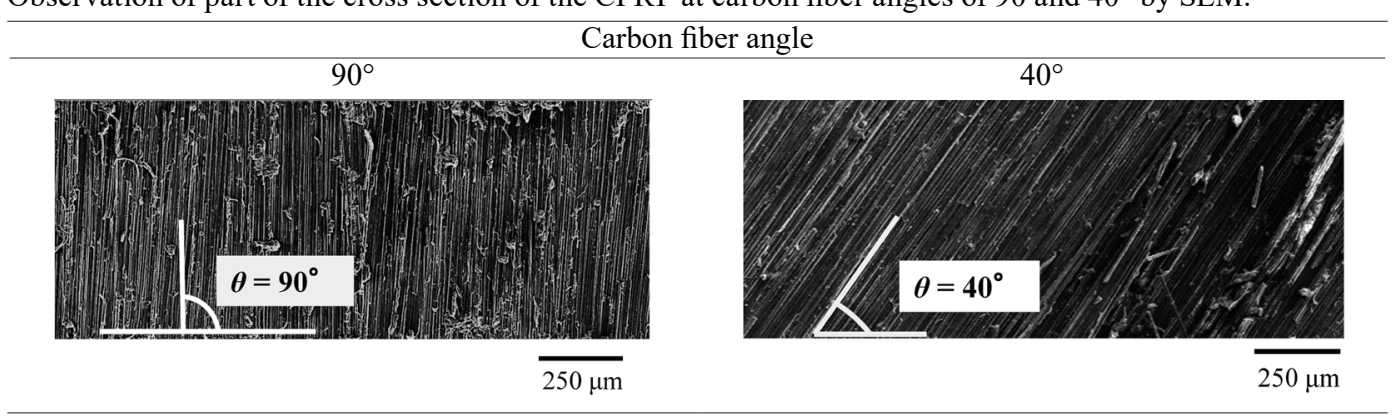



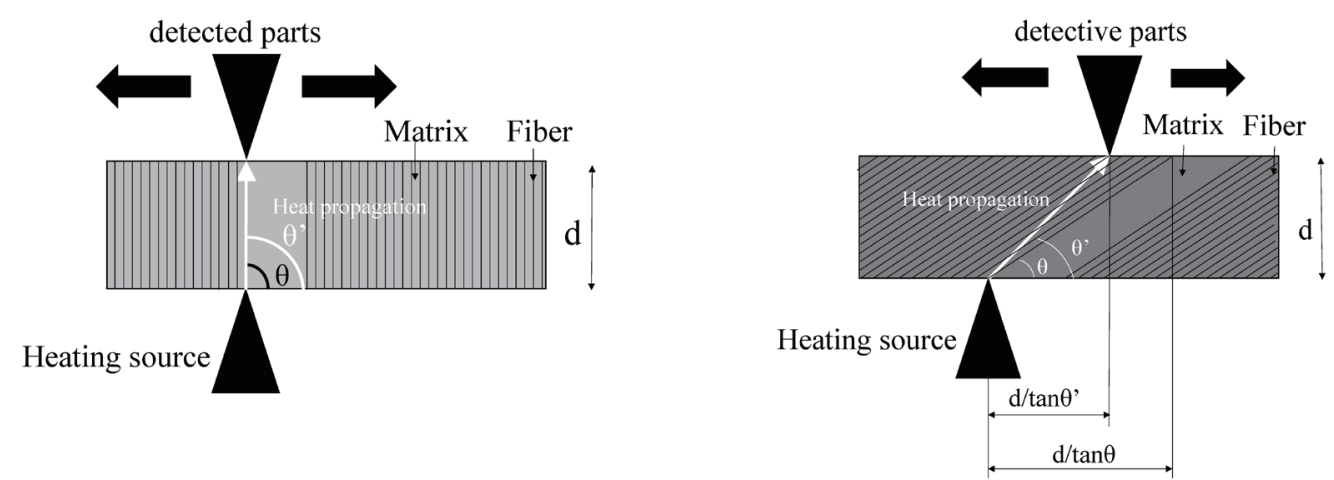

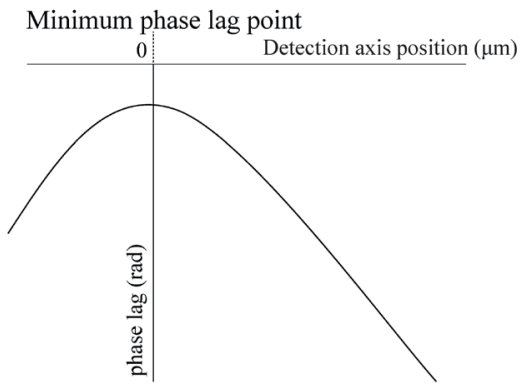

(a)

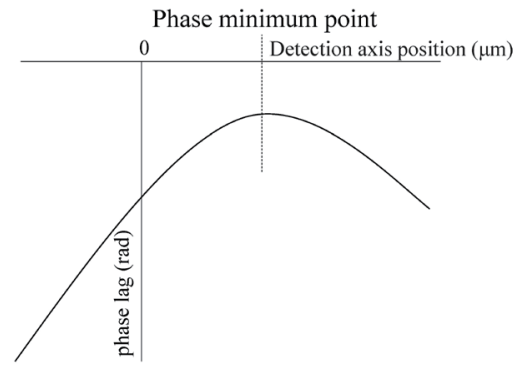

(b)

Fig. 3. Schematic of in-plane measurement and relationship between detection axis position and phase lag in CFRP at fiber angles (a) $\theta=90^{\circ}$ and (b) $\theta \neq 0^{\circ}, 90^{\circ}$.

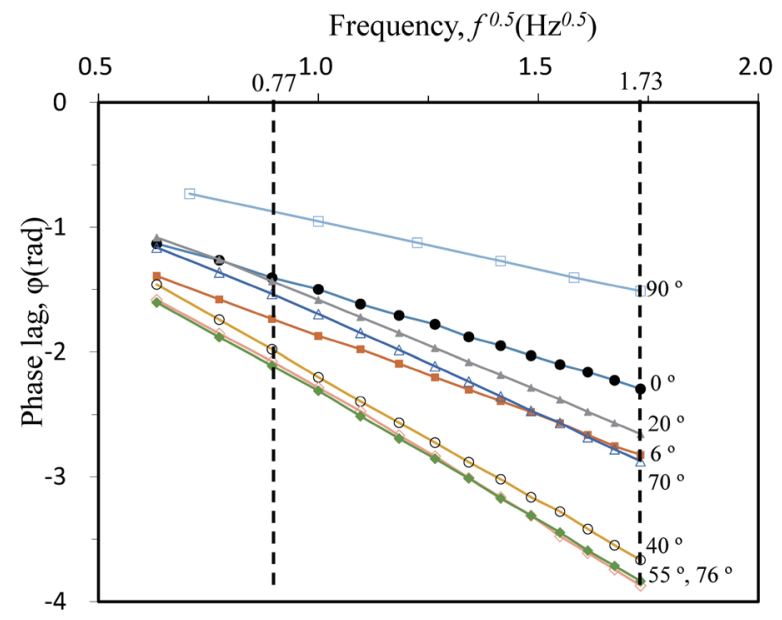

Fig. 4. (Color online) Out-of-plane measurement results for all samples.

$$
r=\frac{d}{\tan \theta^{\prime}},
$$

where $d$ is the thickness of the sample.

Here, to determine the appropriate heating frequency in in-plane measurements by referring to Sect. 2.1, an out-of-plane measurement was conducted for all samples by sweeping the heating 
frequency. The measurement frequency range was set to $0.4-3 \mathrm{~Hz}$ on the basis of Eq. (3), referring to the thermal diffusivity of the CFRP ${ }^{(19)}$ By linear regression, ${ }^{(11,20)}$ the correlation coefficient of the measurement result was estimated as greater than 0.995 . Then, the thermal diffusivity was calculated by considering the slope and heating frequency.

The out-of-plane measurement results of all the samples are shown in Fig. 4. The phase lags to the square root of the frequency are plotted. As one example, in the sample with a carbon fiber angle of $90^{\circ}$, a linear relationship was found within the range of the square root of the frequency from 0.77 to $1.73 \mathrm{~Hz}^{0.5}$. The linearly regressed range was considered an appropriate frequency on the basis of Eq. (3). Since $1 \mathrm{~Hz}$ was within the linearly regressed range in all the samples, the frequency in in-plane-direction measurements was fixed at $1 \mathrm{~Hz}$ in this study.

The in-plane phase lag was measured for all CFRP samples in the scanning range of $\pm 1500 \mu \mathrm{m}$ or -1500 to $2000 \mu \mathrm{m}$ with $50 \mu \mathrm{m}$ steps. If the measurement results are not proportional in the scanning range, the scanning range should be expanded from -1500 to $2000 \mu \mathrm{m}$. The in-plane measurement result was fitted by linear regression, and the thermal diffusivity was calculated from the linear slope and distance between the heating and detecting points.

\section{Results and Discussion}

\subsection{Relationship between fiber and heat propagation angles}

Figure 5 shows the measurement results of the sample at the fiber angles (a) $\theta=90^{\circ}$ and (b) $\theta=40^{\circ} . r$ was obtained from the minimum phase lag point and the heat propagation angle $\theta^{\prime}$ was calculated using $r$ and the thickness $d$ of each sample. In Fig. 5(a), since the heat propagation completely corresponds to the fiber angle, the minimum phase lag point is at the 0 position on the detection axis. The phase lags are shown to be linearly symmetric with respect to the detection axis position line, and linear relationships are observed on the right and left sides of the minimum phase lag point. On the other hand, in Fig. 5(b), since the heat propagation is affected by the inclined carbon fiber, the minimum phase lag point shifts $750 \mu \mathrm{m}$ toward the direction of the inclined carbon

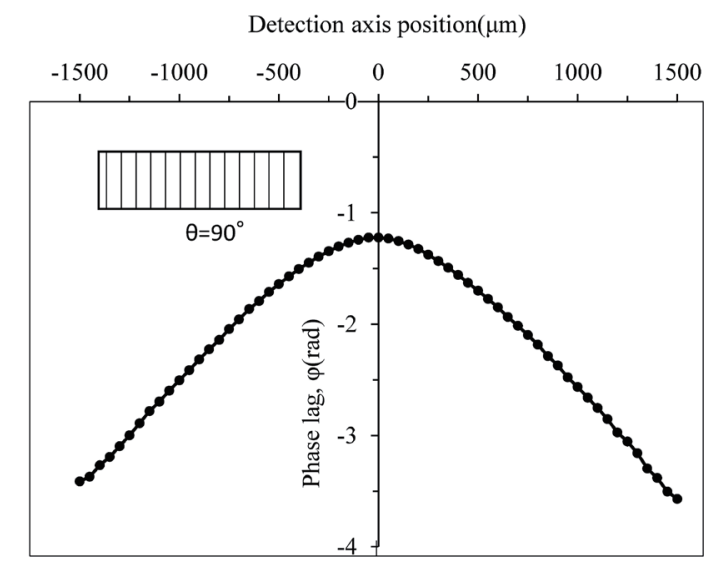

(a)

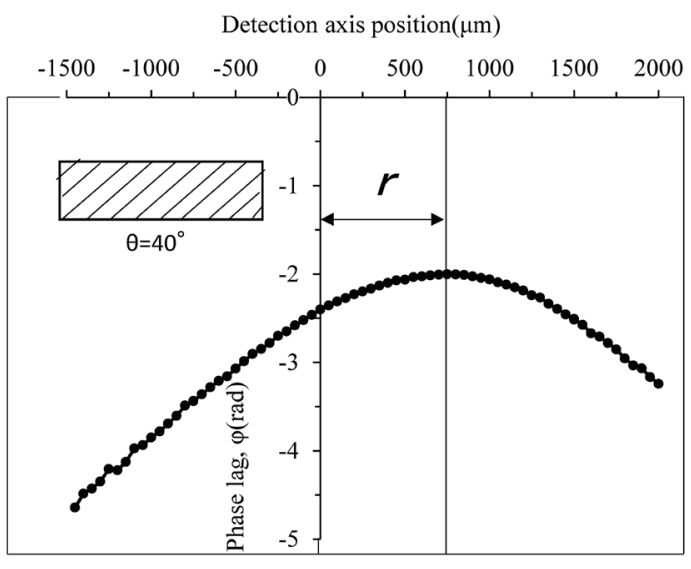

(b)

Fig. 5. In-plane measurement results of the sample at fiber angles (a) $\theta=90^{\circ}$ and (b) $\theta=40^{\circ}$. 
fiber. The heat propagation angle calculated from $d$ and $r$ is $54^{\circ}$. The phase lags are nonsymmetric with respect to the detection axis position line, but linear relationships are observed on the right and left sides of the minimum phase lag point.

Figure 6 shows the relationship between the carbon fiber angle and the heat propagation angle. The broken line indicates that the heat propagation angle $\theta>$ perfectly corresponds to the fiber angle $\theta$. In the fiber angle range from 70 to $90^{\circ}, \theta^{\prime}$ is in good agreement with the broken line. However, $\theta^{\prime}$ gradually diverges at fiber angles below $70^{\circ}$ because the heat flux propagates through the interface between the carbon fibers and the matrix. At the fiber angle of $20^{\circ}$, the smallest $\theta^{\prime}$ of $48.1^{\circ}$ is obtained. At fiber angles of $20^{\circ}$ or less, $\theta>$ increases with decreasing $\theta$ because of the fact that the phase lag of the heat flux through the interface between the carbon fibers and the matrix is mainly detected rather than that into the carbon fibers. Finally, in the case of the fiber angles $\theta=0^{\circ}$ and $\theta^{\prime}=90^{\circ}$, since the heat flux following the carbon fiber is oriented horizontally, information on the heat flux into the carbon fiber cannot be obtained.

Hence, the heat propagation does not completely follow the direction of the carbon fiber in the CFRP and depends on the thermal and physical characteristics of the CFRP.

\subsection{Dependence of thermal diffusivity on carbon fiber angle}

Figure 7 shows the relationship between the carbon fiber angle and the thermal diffusivity of the CFRP samples. The out-of-plane-direction thermal diffusivity increased while approaching the fiber angle in the vertical direction. On the other hand, the in-plane-direction carbon fiber angle tended to be low. This is because the heat propagation angle corresponds to the carbon fiber angle. The out-of-plane thermal diffusivity at the fiber angle of $90^{\circ}$ differs from the in-plane thermal diffusivity at the fiber angle of $0^{\circ}$. We do not know the cause of the divergence, but we believe the measurement to be accurate because the slope of the phase lag used for calculating the thermal diffusivity is linearly regressed with a correlation coefficient of over 0.995 . In the inset of Fig. 7, the in-plane right plots indicate the values of thermal diffusivities in the inclined

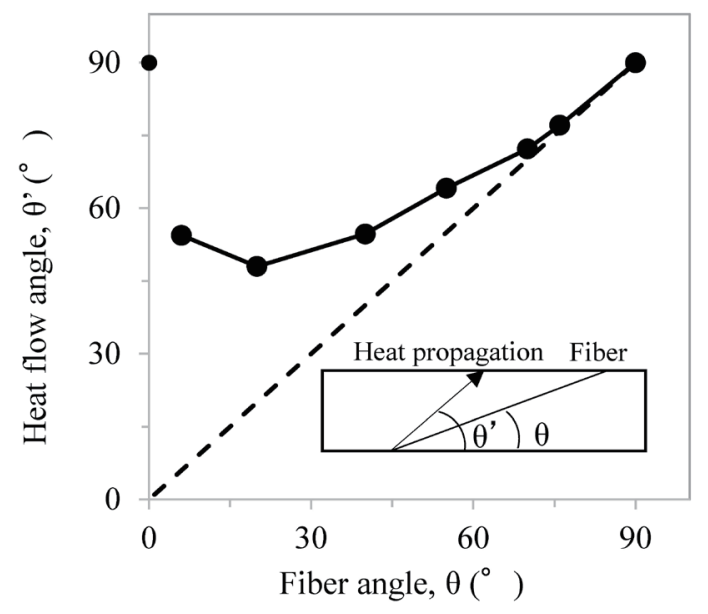

Fig. 6. Relationship between fiber angle and heat propagation angle. 


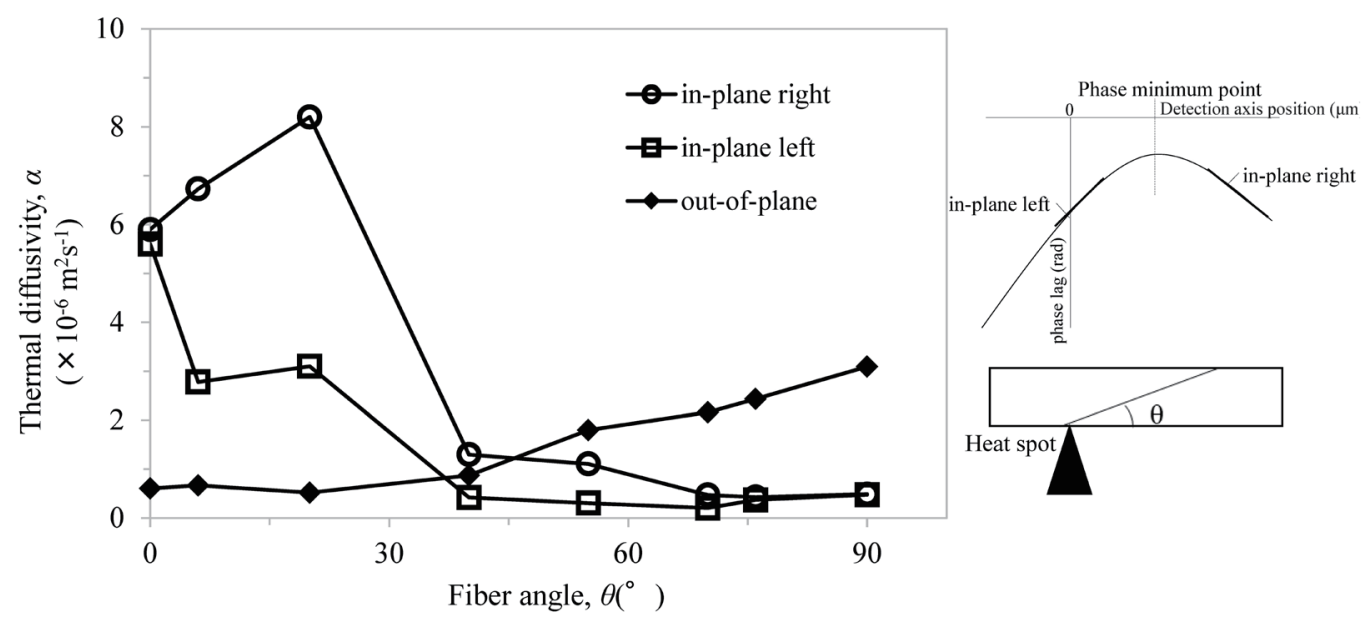

Fig. 7. Relationship between fiber angle and thermal diffusivity.

fiber direction, and the in-plane left plots indicate the thermal diffusivities in the opposite direction. In the fiber angle range excluding 0 and $90^{\circ}$, the in-plane-direction thermal diffusivity differs between in-plane right and in-plane left. Owing to the inclination of the carbon fibers, for in-plane right, the effect of the interface between the carbon fibers and the matrix on the heat propagation is reduced. On the other hand, for in-plane left, it is enhanced. In the case of the fiber angle of $20^{\circ}$, the maximum in-plane thermal diffusivity in all the samples is $8.2 \times 10^{-6} \mathrm{~m}^{2} \mathrm{~s}^{-1}$. Furthermore, the in-plane-direction thermal diffusivity greatly differs between the fiber angles of 40 and $20^{\circ}$. It is likely that in the carbon fiber angle range below $40^{\circ}$, the heat propagation is dominated by the orientation of the carbon fiber in the in-plane direction.

At the fiber angle of $20^{\circ}$, the heat propagation angle is the smallest, as shown in Fig. 6, and the highest thermal diffusivity is obtained in the in-plane direction, as shown in Fig. 7. As a result, the characteristic thermal diffusivity of the CFRP seems to be related to the direction of the heat propagation to the carbon fiber angle. In future works, the cause of the phenomenon observed between the thermal diffusivity and the direction of the heat propagation should be revealed through numerical calculations and measurements with periodic heating.

\section{Conclusions}

We developed a thermal anisotropy estimation method for a fiber-oriented CFRP by analyzing the dependence of measured phase lags on the distance from the heating spot to the detected point using a periodic heating method. In the inclination angle range of the carbon fibers from 70 to $90^{\circ}$, heat propagation is in good agreement with the carbon fiber orientation. By inclining the carbon fiber horizontally, the direction of the heat propagation gradually diverged from the carbon fiber direction. The proposed method might be effective for evaluating the thermal anisotropy of CFRP. Furthermore, as the heat propagation angle corresponds to the fiber angle, thermal diffusivity tended to be high. The carbon fiber orientation affects the thermal diffusivity of the CFRP. In this study, the relationship between the fiber angle and the heat propagation angle can be evaluated by periodic heating. 


\section{Acknowledgments}

We express our gratitude to T. Awano and T. Kato of Bethel Co., Ltd., for their fruitful technical advice regarding the phase lag measurement using the TA.

\section{References}

1 T. Yoneyama: J. Jpn. Soc. Technol. Plast. 55 (2014) 592 (in Japanese).

2 T. Tanaka: Seikei-Kakou 30 (2018) 356 (in Japanese).

3 A. Yamanaka and K. Amaoka: Seikei-Kakou 30 (2018) 406 (in Japanese).

4 K. Uzawa: J. Soc. Mater. Sci. Jpn. 67 (2018) 754 (in Japanese).

5 T. Ono: J. Jpn. Soc. Compos. Mater. 11 (1985) 191 (in Japanese).

6 H. Kim, G. Ben, and Y. Aoki: J. Jpn. Soc. Compos. Mater. 34 (2008) 51 (in Japanese).

7 T. Shimizu, K. Nagai, T. Murai, M. Nakama, and T. Matsuda: J. Jpn. Soc. Compos. Mater. 37 (2011) 21 (in Japanese).

8 D. Wu, K. Sakata, G. Ben, S. Kuraishi, T. Miyauchi, and T. Sonehara: J. Jpn. Soc. Compos. Mater. 40 (2014) 55 (in Japanese).

9 Y. Kageyama: Materia Jpn. 53 (2014) 612 (in Japanese).

10 N. Kurihara and H. Nagano: Netsu Bussei 28 (2015) 82 (in Japanese).

11 A. Tsuji and H. Nagano: Trans. Jpn. Soc. Mech. Eng. 83 (2017) 16 (in Japanese).

12 Y. Kawate, T. Horiuchi, E. Sonoi, and T. Oui: J. Cryo. Soc. Jpn. 14 (1979) 164 (in Japanese).

13 M. Takeno, S. Nishijima, T. Okata, and Y. Kuraoka: J. Cryo. Soc. Jpn. 21 (1986) 182 (in Japanese).

14 H. Kato: Netsu Bussei 15 (2001) 95 (in Japanese).

15 H. Kato, T. Baba, and M. Okaji: Meas. Sci. Technol. 12 (2001) 2074.

16 Y. Kawahara, H. Iwata, H. Hagino, and K. Miyazaki: Trans. Jpn. Soc. Mech. Eng. 66 (2013) 19 (in Japanese).

17 H. S. Carslaw and J. C. Jaeger: Conduction of Heat in Solids (Oxford Science Publications, Oxford, 1959) p. 26.

18 M. Kaneaki, H. Saito, M. Tanaka, M. Hojo, and I. Kimpara: J. Jpn. Soc. Compos. Mater. 39 (2013) 89 (in Japanese).

19 Y. Tanabe, S. Kimura, and E. Yasuda: J. Ceram. Soc. Jpn. 95 (1987) 341 (in Japanese).

20 O. Pessoa Jr., C. L. Cesar, N. A. Patel, and H. Vargas: J. Appl. Phys. 59 (1986) 1316. 